\title{
EXTENDED CENTROIDS OF POWER SERIES RINGS
}

\author{
by W. S. MARTINDALE, M. P. ROSEN and J. D. ROSEN
}

(Received 19 June, 1989; revised 10 November, 1989)

Prime rings came into prominence when Posner characterized prime rings satisfying a polynomial identity [9]. The scarcity of invertible central elements made it difficult to generalize results from central simple and primitive algebras to prime rings. For example, we do not automatically have tensor products at our disposal. In [5], the first author introduced the Martindale ring of quotients $Q(R)$ of a prime ring $R$ in his theorem characterizing prime rings satisfying a generalized polynomial identity (GPI). $Q(R)$ is a prime ring containing $R$ whose center $C$ is a field called the extended centroid of $R$. The central closure of $R$ is the subring $R C$ of $Q(R)$ generated by $R$ and $C$. $R C$ is a closed prime ring since its extended centroid equals its center $C$. Hence we have a useful procedure for proving results about an arbitrary prime ring $R$. We first answer the question for closed prime rings and then apply to $R$ the information obtained from $R C$. It should be noted that simple rings and free algebras of rank at least 2 are closed prime rings. For these reasons, closed prime rings are natural objects to study.

The extended centroid has been computed for many classes of rings, such as some group rings [2], skew polynomial rings of automorphism and derivation type [7], [10], coproducts of algebras, and free algebras [6]. The ring of quotients $Q(R)$ and its various subrings have had numerous applications. As mentioned above, they played a key role in the solution of the GPI problem. In [4], Kharchenko developed a Galois theory of semiprime rings which makes extensive use of these ideas. We refer the reader to [8] for a simplified account of this work over prime rings.

In this paper, we determine the extended centroid of the power series ring $R[[x]]$ over a closed prime ring $R$. One motivation for this problem comes from commutative algebra. In this case, finding the extended centroid of a prime ring is equivalent to finding its field of quotients. If $F$ is a field, then the field of quotients of $F[[x]]$ is $F((x))$, the field of Laurent series over $F$. However, if $D$ is an integral domain then the field of quotients of $D[[x]]$ is not known in general. In particular, it is rarely the case that the field of quotients of $D[[x]]$ is $F((x))$, where $F$ is the field of quotients of $D$. We will provide an example where $D=\mathbb{Z}$ (the integers) and refer the reader to [1], [3] for more general examples. If $R$ is a closed prime ring over $C$, we prove the extended centroid of $R[[x]]$ is $C((x))$. This theorem generalizes the corresponding result for $F[[x]]$. As a corollary, we show that if $R$ is a closed prime ring then so is the ring of Laurent series $R((x))$.

1. Preliminaries. Let $R[[x]]$ denote the power series ring over $R$. If $f=\sum_{i=0}^{\infty} r_{i} x^{i}$ is a nonzero power series in $R[[x]]$ then the degree of $f$, denoted $\operatorname{deg}(f)$, is the nonnegative integer $n$ such that $r_{i}=0$ for $i<n$ and $r_{n} \neq 0$. Let $R((x))$ denote the ring of Laurent series over $R$, i.e.

$$
R((x))=\left\{\sum_{i=n}^{\infty} r_{i} x^{i} \mid r_{i} \in R, n \in \mathbb{Z}\right\} .
$$

If $R$ is a prime ring then $R[[x]]$ and $R((x))$ are also prime.

Glasgow Math. J. 32 (1990) 371-375. 
We now summarize the definition of the extended centroid of a prime ring $R$ with 1 . We refer the reader to [5] for a more detailed account. Let $\mu=\{U\}$ be the collection of all nonzero two-sided ideals of $R$ and consider the totality $T$ of all right module homomorphisms $\phi: U_{R} \rightarrow R_{R}$, where $U \in \mu$ and $U$ and $R$ are regarded as right $R$-modules. Write $(\phi, U)$ for an element of $T$ and define an equivalence relation $\sim$ on $T$ as follows: $(\phi, U) \sim(\psi, V)$ if $\phi=\psi$ on some $W \in \mu$, where $W \subseteq U \cap V$. Let $(\overline{\phi, U})$ denote the equivalence class of $(\phi, U)$. The Martindale ring of quotients $Q(R)$ is defined to be the set of these equivalence classes. $Q(R)$ is made into a ring as follows:

$$
\begin{gathered}
(\overline{\phi, U})+(\overline{\psi, V})=(\overline{\phi+\psi, U \cap V}) \\
(\overline{\phi, U})(\overline{\psi, V})=(\overline{\phi \circ \psi, V U}) \quad \text { (composition acting on the left). }
\end{gathered}
$$

$R$ may be considered a subring of $Q(R)$ via the mapping $a \rightarrow\left(\overline{a_{l}, R}\right)$, where $a_{l}$ is the left multiplication by $a$ acting on $R . Q(R)$ is a prime ring whose center $C$ is a field called the extended centroid of $R$. The pair $(\phi, U)$ is permissible if $\phi:{ }_{R} U_{R} \rightarrow{ }_{R} R_{R}$ is a bimodule homomorphism. $C$ may also be characterized as the set

$$
\{(\overline{\phi, U}) \in Q(R) \mid(\phi, U) \text { is permissible }\} \text {. }
$$

We may now form the central closure $R C$ of $R$. $R C$ is a prime ring with center $C$. A prime algebra over a field $F$ is said to be closed if $F$ is already its extended centroid. Note that $R C$ is closed over $C$.

Lemma 1.1. Let $R$ be a prime ring with center $Z(R)$ and extended centroid $C$. Let $F$ be the field of fractions of $Z(R)$. Suppose for any $(\overline{\phi, U}) \in C$, there exists nonzero $u \in U$ such that $\phi(u)=\alpha u$, where $\alpha \in F$. Then $C$ is isomorphic to $F$.

Proof. Set $\lambda=(\overline{\phi, U})$. Thus $\phi(u)=\lambda u=\alpha u$, implying $(\lambda-\alpha) u=0$. Viewing this equation in $R C$, we see that $\lambda=\alpha$.

EXAmple. We claim that $\mathbb{Q}((x))$ is not the extended centroid of $\mathbb{Z}[[x]]$, where $\mathbb{Z}$ and $\mathbb{Q}$ denote the integers and rationals respectively.

Let $f=x+\frac{1}{2} x^{2}+\frac{1}{3} x^{3}+\ldots$ It suffices to show that $f g$ is not in $\mathbb{Z}[[x]]$ for any nonzero $g \in \mathbb{Z}[[x]]$. Suppose otherwise with $g=\sum_{i=n}^{\infty} a_{i} x^{i}$. Thus for any prime $p$, the coefficient of $x^{n+p}$ in $f g$ is $a_{n+p-1}+\frac{1}{2} a_{n+p-2}+\ldots+\frac{1}{p-1} a_{n+1}+\frac{1}{p} a_{n} \in \mathbb{Z}$. Multiplying by $(p-1)$ !, we can see that $p \mid a_{n}$, a contradiction.

2. Main theorem. In the following four results, let $R$ be a prime ring with extended centroid $C$. Let $D$ denote the extended centroid of $R[[x]]$ and assume $(\overline{\phi, U}) \in D$, $(\overline{\phi, U}) \neq 0$. We remark that $\phi$ is one-to-one on $U$.

Lemma 2.1. If $f, g \in U, f, g \neq 0$ then $\operatorname{deg}(f)+\operatorname{deg}(\phi(g))=\operatorname{deg}(g)+\operatorname{deg}(\phi(f))$.

Proof. Let $f=a x^{n}+\ldots$ and $g=b x^{k}+\ldots$, where $a, b \neq 0$. Set $\phi(f)=c x^{m}+\ldots$ and $\phi(g)=d x^{l}+\ldots$, where $c, d \neq 0$. Assume $k+m<n+l$. Since $R$ is prime, there 
exists $r \in R$ such that $c r b \neq 0$. Since $\phi$ is bimodule,

$$
\phi(f r g)=\left(c x^{m}+\ldots\right) r\left(b x^{k}+\ldots\right)=\left(a x^{n}+\ldots\right) r\left(d x^{l}+\ldots\right) .
$$

Equating coefficients, we obtain $c r b=0$, a contradiction. Similarly $n+l \geq k+m$.

The next two corollaries are immediate consequences of Lemma 2.1.

Corollary 2.2. If $f \in U$ is of minimal degree then $\phi(f)$ is of minimal degree in $\phi(U)$.

CoROllary 2.3. If there exists $f \in U$ such that $\operatorname{deg}(f)=\operatorname{deg}(\phi(f))=0$ then $\operatorname{deg}(g)=0$ implies $\operatorname{deg}(\phi(g))=0$ for $g \in U$.

Corollary 2.4. Suppose $g \in U$ such that $\operatorname{deg}(g)=\operatorname{deg}(\phi(g))=0$. If $a_{0}, b_{0}$ are the degree 0 coefficients of $g$ and $\phi(g)$ respectively then there exists $\lambda \in C$ such that $b_{0}=\lambda a_{0}$.

Proof. $I=\{0\} \cup\{r \in R \mid r$ is the degree 0 coefficient of some element in $U\}$ is a nonzero ideal of $R$. Choose $r \neq 0$ in $l$ and suppose $r+r_{1} x+\ldots$ and $r+s_{1} x+\ldots$ are distinct elements of $U$. Let

$$
\phi\left(r+r_{1} x+\ldots\right)=t_{0}+t_{1} x+\ldots \text { and } \phi\left(r+s_{1} x+\ldots\right)=u_{0}+u_{1} x+\ldots
$$

By Corollary $2.3, t_{0}, u_{0} \neq 0$. Applying Lemma 2.1 to $g$ and $\left(r_{1}-s_{1}\right) x+\ldots$, we get $t_{0}=u_{0}$. Define $\lambda: I \rightarrow R$ by $0 \rightarrow 0$ and $r \rightarrow t$, where $t$ is the unique degree 0 coefficient of the image under $\phi$ of any element in $U$ having $r$ as its degree 0 coefficient. $\lambda$ is a bimodule homomorphism. Hence $\lambda \in C$ and $b_{0}=\lambda a_{0}$.

The following theorem is the key result needed for determining $D$ in the case where $R$ is closed prime.

THeOREM 2.5. Let $R$ be a closed prime ring with extended centroid $C$. Let $D$ denote the extended centroid of $R[[x]]$ and suppose $(\overline{\phi, U}) \in D$. Then there exists nonzero $f \in U$ such that $\phi(f)=h f$, where $h \in C((x))$.

Proof. Choose $f \in U$ of minimal degree $n$ and let $m=\operatorname{deg}(\phi(f)) . V=x^{-n} U$ is a nonzero ideal of $R[[x]]$. By Corollary $2.2,\left(\overline{\eta_{0}, V}\right) \in D$, where $\eta_{0}\left(x^{-n} k\right)=x^{-m} \phi(k)$ for all $k \in U$. Setting $g=x^{-n} f$, we have $\operatorname{deg}(g)=\operatorname{deg}\left(\eta_{o}(g)\right)=0$. Let

$$
g=\sum_{i=0}^{\infty} a_{i} x^{i} \text { and } \eta_{0}(g)=\sum_{i=0}^{\infty} b_{i} x^{i}
$$

We use induction to find $\lambda_{i} \in C$ such that

$$
b_{i}=\lambda_{0} a_{i}+\lambda_{1} a_{i-1}+\ldots+\lambda_{i} a_{0} \text { for all } i \text {. }
$$

By Corollary 2.4, there exists $\lambda_{0} \in C$ such that $b_{0}=\lambda_{0} a_{0}$. Since $R$ is closed prime, $\lambda_{0}$ acts on $R$ and hence on $R[[x]]$ as left multiplication. We claim that $\eta_{1}=x^{-1}\left(\eta_{0}-\lambda_{0}\right)$ acts on $W=R[[x]] g R[[x]]$. It suffices to apply $\eta_{1}$ to $p g q$, where $p, q \in R[[x]]$. Now

$$
\begin{aligned}
\eta_{1}(p g q) & =x^{-1}\left(p \eta_{0}(g) q-p \lambda_{0} g q\right) \\
& =x^{-1}\left(p\left(\lambda_{0} a_{0}+b_{1} x+\ldots\right) q-p\left(\lambda_{0} a_{0}+\lambda_{0} a_{1} x+\ldots\right) q\right) \in R[[x]] .
\end{aligned}
$$

Thus $\left(\overline{\eta_{1}, W}\right) \in D$. In particular, $\eta_{1}(g)=\left(b_{1}-\lambda_{0} a_{1}\right)+\left(b_{2}-\lambda_{0} a_{2}\right) x+\ldots$ If $b_{1}-\lambda_{0} a_{1}=$ 0 , choose $\lambda_{1}=0$. Otherwise, by Corollary 2.4 , there exists $\lambda_{1} \in C$ such that $b_{1}-\lambda_{0} a_{1}=\lambda_{1} a_{0}$ 
or $b_{1}=\lambda_{0} a_{1}+\lambda_{1} a_{0}$. Assume there exist $\lambda_{2}, \ldots, \lambda_{i-1} \in C$ and corresponding $\eta_{2}, \ldots, \eta_{i-1}$ (acting on $W$ ) such that

and

$$
b_{i-1}=\lambda_{0} a_{i-1}+\lambda_{1} a_{i-2}+\ldots+\lambda_{i-1} a_{0}
$$

$$
\begin{aligned}
\eta_{i-1}(g)=\left(b_{i-1}-\lambda_{0} a_{i-1}-\lambda_{1} a_{i-2}-\ldots-\right. & \left.\lambda_{i-2} a_{1}\right) \\
& +\left(b_{i}-\lambda_{0} a_{i}-\lambda_{1} a_{i-1}-\ldots-\lambda_{i-2} a_{2}\right) x+\ldots .
\end{aligned}
$$

Let $\eta_{i}=x^{-1}\left(\eta_{i-1}-\lambda_{i-1}\right)$. Note that $\left(\overline{\eta_{i}, W}\right) \in D$ and the degree 0 coefficient of $\eta_{i}(g)$ is $b_{i}-\lambda_{0} a_{i}-\lambda_{1} a_{i-1}-\ldots-\lambda_{i-1} a_{1}$. If this term is 0 , choose $\lambda_{i}=0$. Otherwise, by Corollary 2.4 , there exists $\lambda_{i} \in C$ such that $b_{i}=\lambda_{0} a_{i}+\ldots+\lambda_{i} a_{0}$. Thus

$$
\begin{aligned}
\eta_{0}(g)=\lambda_{0} a_{0}+\left(\lambda_{0} a_{1}+\lambda_{1} a_{0}\right) x+\left(\lambda_{0} a_{2}+\lambda_{1} a_{1}+\lambda_{2} a_{0}\right) x^{2}+\ldots & \\
& =\left(\lambda_{0}+\lambda_{1} x+\lambda_{2} x^{2}+\ldots\right) g .
\end{aligned}
$$

Recall that $g=x^{-n} f$ and $\eta_{0}(g)=x^{-m} \phi(f)$. Therefore

$$
x^{-m} \phi(f)=\left(\lambda_{0}+\lambda_{1} x+\ldots\right) x^{-n} f \text { or } \phi(f)=\left(\lambda_{0}+\lambda_{1} x+\ldots\right) x^{m-n} f=h f \text {, }
$$

where $h \in C((x))$.

By Lemma 1.1 and Theorem 2.5, we obtain the main result of this paper.

THEOREM 2.6. Let $R$ be a closed prime ring with extended centroid $C$. Then the extended centroid of $R[[x]]$ is isomorphic to $C((x))$.

Note that the example in Section 1 shows Theorem 2.6 fails if $R$ is not closed. We end with two results on the extended centroid of $R((x))$.

THEOREM 2.7. For any prime ring $R, R[[x]]$ and $R((x))$ have isomorphic extended centroids.

Proof. Let $D$ and $E$ denote the extended centroids of $R[[x]]$ and $R((x))$ respectively. Choose $(\overline{\phi, U}) \in D$ and define $U^{\prime}=\left\{x^{-m} f \mid f \in U, m \geq 0\right\}$. If $x^{-m} f, x^{-n} g \in U^{\prime}$ with $m>n$ then $x^{-m} f+x^{-n} g=x^{-m}\left(f+x^{m-n} g\right) \in U^{\prime}$. If $h \in R((x))$ such that $h=x^{-l} k$, where $l \geq 0$ and $k \in R[[x]]$, then $x^{-m} f h=x^{-(m+l)} f k \in U^{\prime}$. Hence $U^{\prime}$ is a nonzero ideal of $R((x))$. Define $\phi^{\prime}: U^{\prime} \rightarrow R((x))$ by $\phi^{\prime}\left(x^{-m} f\right)=x^{-m} \phi(f)$. If $x^{-m} f=x^{-n} g$ with $m>n$ then $\phi(f)=$ $x^{m-n} \phi(g)$, which shows that $x^{-m} \phi(f)=x^{-n} \phi(g)$. Using the above computations, one can verify that $\left(\overline{\phi^{\prime}, U^{\prime}}\right) \in E$. The mapping $(\overline{\phi, U}) \rightarrow\left(\overline{\phi^{\prime}, U^{\prime}}\right)$ gives the desired isomorphism from $D$ to $E$. The fact that every nonzero ideal of $R((x))$ intersects $R[[x]]$ nontrivially implies this mapping is onto.

CoROllary 2.8. Let $R$ be a closed prime ring with extended centroid $C$. Then $R((x))$ is closed prime with extended centroid $C((x))$.

\section{REFERENCES}

1. J. W. Brewer, Power series over commutative rings, Lecture Notes in Pure and Appl. Math. 64 (Marcel Dekker, 1981).

2. E. Formanek, Maximal quotient rings of group rings, Pacific J. Math. 53 (1974), 109-116. 
3. R. Gilmer, Multiplicative ideal theory, Pure Appl. Math. 12 (Marcel Dekker, 1972).

4. V. K. Kharchenko, Galois theory of semiprime rings, Algebra i Logika 16 (1977), 313-363; English transl. (1978), 208-258.

5. W. S. Martindale, Prime rings satisfying a generalized polynomial identity, J. Algebra 12 (1969), 576-584.

6. W. S. Martindale, The normal closure of the coproduct of rings over a division ring, Trans. Amer. Math. Soc. 293 (1986), 303-317.

7. J. Matczuk, Extended centroids of skew polynomial rings, Math. J. Okayama Univ. 30 (1988), 13-20.

8. D. S. Passman, Infinite cross products, Pure Appl. Math. 135 (Academic Press, 1989).

9. E. C. Posner, Prime rings satisfying a polynomial identity, Proc. Amer. Math. Soc. 11 (1960), 180-184.

10. J. Rosen and M. Rosen, Extended centroids of skew polynomial rings, Canad. Math. Bull. 28 (1985), 67-76.

Department of Mathematics

University of MassachusetTs at AMHerst

AmHerst, MassachusetTs 01003
DePaRTMENT OF MATHEMATICS

California State University, Northridge Northridge, California 91330 